Pacific Journal of Mathematics

ON THE SATO-SEGAL-WILSON SOLUTIONS OF THE K-DV

EQUATION 


\title{
ON THE SATO-SEGAL-WILSON SOLUTIONS OF THE K-dV EQUATION
}

\author{
RusSELl A. JoHNSON
}

\begin{abstract}
We discuss the class of solutions of the K-dV equation found by Sato, Segal, and Wilson. We relate this class of solutions to properties of the Weyl $m$-functions, and of the Floquet exponent for the random Schrödinger equation.
\end{abstract}

1. Introduction. In a series of recent papers, Date, Jimbo, Kashiwara, and Miwa $[\mathbf{5}, \mathbf{6}, \mathbf{7}, \mathbf{8}, \mathbf{9}]$ have developed ideas of $M$. and Y. Sato $[\mathbf{2 3}, \mathbf{2 4}]$ for finding solutions of the Kadomtsev-Petviashvili (K-P) hierarchy. The solutions of the K-P hierarchy discussed in these papers are expressed in terms of the so-called $\tau$-function, which can be viewed as a generalization of the Riemann $\Theta$-function.

Even more recently, Segal and Wilson [25] have given a careful formulation of the work of the Kyoto group. A consequence of their analysis is the following. Recall that one equation of the K-P hierarchy is the Korteweg-de Vries (K-dV) equation:

$$
\frac{\partial u}{\partial t}=6 u \frac{\partial u}{\partial x}-\frac{\partial^{3} u}{\partial x^{3}}, \quad u(0, x)=u_{0}(x),
$$

viewed as an evolution equation with initial data $u_{0}(x)$. Segal and Wilson produce a class $\mathscr{C}^{(2)}$ of initial conditions (or "potentials") $u_{0}(x)$ for which (1) admits a solution $u(t, x)$ which is meromorphic in $t$ and $x$. The class $\mathscr{C}^{(2)}$ contains the solitons (see, e.g., [1]) and the algebro-geometric potentials $[\mathbf{1 1}, \mathbf{1 8}, \mathbf{2 1}]$. We will call the elements of $\mathscr{C}^{(2)}$ Sato-Segal-Wilson potentials.

The purpose of the present note is to describe in some detail a subclass LP (for "limit-point"; see below) of the class $\mathscr{C}^{(2)}$. Namely, consider the Schrödinger equation

$$
L \phi=\left(\frac{-d^{2}}{d x^{2}}+u_{0}(x)\right) \phi=\lambda \phi
$$

with potential $u_{0}(x)$. Define $\mathrm{LP} \subset \mathscr{C}^{(2)}$ to be the set of Sato-SegalWilson potentials which are real and finite for all real $x$, and for which $L$ is in the limit-point case $x= \pm \infty$ ([26]; [3, Ch. 9]). Let $m_{+}(\lambda)$ be the 
corresponding Weyl $m$-functions; they are defined and holomorphic for $\operatorname{Im} \lambda \neq 0$. Define

$$
\mathscr{M}(z)= \begin{cases}m_{+}\left(z^{2}\right), & \operatorname{Im} z>0, \operatorname{Re} z \neq 0, \\ m_{-}\left(z^{2}\right), & \operatorname{Im} z<0, \operatorname{Re} z \neq 0 .\end{cases}
$$

We show that, if $u_{0}$ is in LP, then there exists $r>0$ such that $\mathscr{M}$ extends to a holomorphic function on $|z|>r$ with a simple pole at $z=\infty$. Conversely, if $u_{0}(x)$ is a locally-integrable, real function of $x \in \mathbf{R}$ such that $L=-d^{2} / d x^{2}+u_{0}(x)$ is in the limit-point case at $x= \pm \infty$, and if $m_{ \pm}(\lambda)$ form branches of a function $\mathscr{M}(z)\left(z^{2}=\lambda\right)$ which is holomorphic for $|z|>r$, then $u_{0} \in \mathrm{LP}$.

We use this observation to find $u_{0} \in$ LP for which the spectrum $\Sigma$ of $L$ has a Cantor-like part, i.e. $\Sigma \cap\left(-\infty, r^{2}\right)$ is a Cantor set for some $r \in \mathbf{R}$. We then show how to "explicitly" construct a large subclass of LP. To do so, we use the Floquet exponent $w=w(\lambda)(\operatorname{Im} \lambda \geq 0)$ introduced by Johnson-Moser [15] and studied by Kotani [16, 17], De Concini-Johnson [10], Giachetti-Johnson [13], and others. The construction goes as follows. Let $h(\lambda)$ be a function holomorphic in the upper half-plane $U=\{\lambda \mid \operatorname{Im} \lambda>0\}$ with positive imaginary part and with certain additional properties; in particular it is supposed that the boundary value $\hat{h}(\lambda)=\lim _{\varepsilon \rightarrow 0^{+}} \hat{h}(\lambda+i \varepsilon)(\lambda \in \mathbf{R})$ satisfies $\operatorname{Re} \hat{h}(\lambda)=0$ for large real $\lambda$. In [17], Kotani shows how to find a stationary stochastic process $(\Omega, \mathscr{B}, \mu)$ which (with slight abuse of terminology; see §3) has Floquet exponent $w(\lambda)=h(\lambda)$. By Kotani's construction, $\Omega$ is a subset of a certain Hilbert space of potentials $u_{0}$. It turns out that $\mu$-a.a. potentials are in LP.

Our results may be summarized as follows. On the one hand, potentials in the class LP $\subset \mathscr{C}^{(2)}$ are quite special: the restriction on the behavior of the $m$-functions is very strong. On the other hand, it will be clear from $\$ 3$ that LP contains much more than the solitons and the algebro-geometric potentials.

2. The $m$-functions. We begin with a brief outline of the Segal-Wilson construction of the class $\mathscr{C}^{(2)}$. The formulas below differ slightly from those of [25], because we use $L=-d^{2} / d x^{2}+u_{0}(x)$ instead of $L=$ $+d^{2} / d x^{2}+u_{0}(x)$.

Let $\mathbf{K}$ be the unit circle, and let $H_{+} \subset L^{2}(\mathbf{K})$ be the set of boundary values in $L^{2}(\mathbf{K})$ of holomorphic functions on the unit disc $\{z|| z \mid<1\}$. Thus $H_{+}=$cls $\operatorname{span}\left\{1, z, z^{2}, \ldots\right\}$. One considers subspaces $W \subset L^{2}(\mathbf{K})$ which are comparable with $H_{+}$in the sense that: (i) the orthogonal projection $\operatorname{pr}=\operatorname{pr}(W): W \rightarrow H_{+}$is Fredholm of index zero; (ii) the 
orthogonal projection from $W$ onto $H_{-}=\left(H_{+}\right)^{\perp}=\operatorname{cls} \operatorname{span}\left\{z^{-1}, z^{-2}, \ldots\right\}$ is compact. The group $\Gamma_{+}$of exponential power series

$$
\exp \left(x z+t_{2} z^{2}+t_{3} z^{3}+\cdots\right) \quad\left(x, t_{i} \in \mathbf{C}\right)
$$

acts on the Grassmannian $\mathrm{Gr}$ of all such subspaces $W$ by pointwise multiplication of functions. One constructs a determinant bundle Det over $\mathrm{Gr}$, which in turn can be used to define the determinant of $\operatorname{pr}(W)$ when $W \in \mathrm{Gr}$. The $\tau$-function $\tau_{W}$ of $W$ is now defined as follows:

$$
\tau_{W}\left(x, t_{2}, t_{3}, \ldots\right)=\operatorname{det} \operatorname{pr}(W) / \operatorname{det} \operatorname{pr}\left[\exp \left(-x z-t_{2} z^{2}-\cdots\right) \cdot W\right] \text {. }
$$

Then $\tau_{W}$ is meromorphic in all variables. Moreover if $\operatorname{det} \operatorname{pr}(W) \neq 0$, then $\tau_{W}\left(x, t_{2}, t_{3}, \ldots\right)=\infty$ exactly when $\operatorname{det} \operatorname{pr}\left[\exp \left(-x z-t_{2} z^{2}-\cdots\right) \cdot W\right]=$ 0 , and this occurs exactly when $\exp \left(-x z-t_{2} z^{2}-\cdots\right) \cdot W$ intersects $H_{-}$ nontrivially.

One says that a subspace $W \in \mathrm{Gr}$ is transverse if $W \cap H_{-}=\{0\}$; thus $W$ is transverse iff $\operatorname{det} \operatorname{pr}(W) \neq 0$. The poles of $\tau_{W}$ are in $1-1$ correspondence with non-transverse subspaces $\exp \left(-x z-t_{2} z^{2}-\cdots\right) \cdot W$ if $W$ itself is transverse.

Let us now restrict attention to the subset $\mathrm{Gr}^{(2)}$ of $\mathrm{Gr}$ consisting of subspaces $W \subset L^{2}(\mathbf{K})$ which are invariant under $z^{2}: z^{2} W \subset W$. The subset $\left\{\exp \sum_{i=1}^{\infty} t_{2 l} z^{2 l}\right\}$ of $\Gamma_{+}$leaves such a $W$ fixed. Let $\tau_{W}\left(x, t_{3}, t_{5}, \ldots\right)$ be the corresponding $\tau$-function. Define

$$
u_{W}(x, t)=-2 \frac{d^{2}}{d x^{2}} \log \tau_{W}(i x,-i t, 0,0, \ldots) ;
$$

i.e., $t_{3}=i t$ and all other $t_{i} \mathrm{~s}$ equal zero. Then $u_{W}(x, t)$ is the solution to the K-dV equation (2) with initial condition $u_{0}(x)=u_{W}(x, 0)$.

An important intermediate step in showing that $u_{W}(x, t)$ solves the $\mathrm{K}-\mathrm{dV}$ equation is the construction of the Baker function $\psi_{W}(x, z)$. For our purposes, the following description of $\psi_{W}$ will suffice; a more general discussion is given in $[25, \S 5]$.

Let $W \in \mathrm{Gr}^{(2)}$ be a transverse space, and suppose that $(\exp -i x z) \cdot W$ is transverse for all real $x$. Then there is a unique function

$$
\psi_{W}(x, z)=e^{i x z}\left(1+\sum_{i=1}^{\infty} a_{i}(x) z^{-i}\right)
$$

in the space $W$; in fact $\exp (-i x z) \psi_{W}(x, z)$ is the inverse image of 1 under the orthogonal projection of $\exp (-i x z) \cdot W$ onto $H_{+}$. The series in parentheses converges for $|z|>1$. Moreover

$$
\left(\frac{-d^{2}}{d x^{2}}+u_{0}(x)\right) \psi_{W}(x, z)=z^{2} \psi_{W}(x, z) \quad(x \in \mathbf{R},|z|>1),
$$


where $u_{0}(x)=-2\left(d^{2} / d x^{2}\right) \log \tau_{W}(i x, 0,0, \ldots)$. One calls $\psi_{W}(x, z)$ the Baker function of $W$, or of $u_{0}(x)$.

Note that any differential operator $L=\left(-d^{2} / d x^{2}\right)+u_{0}(x)$ with $C^{\infty}$ potential $u_{0}(x)$ gives rise to a formal Baker function

$$
\tilde{\psi}(x, z)=e^{i x z}\left(1+\sum_{i=1}^{\infty} \tilde{a}_{i}(x) z^{-i}\right)
$$

which formally satisfies (i) $L \tilde{\psi}=z^{2} \tilde{\psi}$, and (ii) $\tilde{\psi}(0, z)=1$. In fact, the coefficients $\tilde{a}_{i}(x)$ are $C^{\infty}$ functions which are determined recursively by $a_{0} \equiv 1, a_{i+1}^{\prime}=(-i / 2) L a_{i}, a_{i}(0)=0(i \geq 1)$. The quantity $e^{-i x z} \tilde{\psi}(x, z)$ is the only element of the ring $\mathscr{L}$ of formal Laurent series $s(x, z)=$ $\sum_{l=1}^{\infty} b_{l}(x) z^{-i}$ with $C^{\infty}$ coefficients $b_{i}(x)$ such that $e^{i x z} s(x, z)$ satisfies (i) and (ii).

Define $\mathscr{C}^{(2)}$ to be the class of (real or complex) potentials $u_{0}(x)$ such that, for some complex $\lambda \neq 0$, there exists $W \in \mathrm{Gr}^{(2)}$ such that $\lambda^{2} u_{0}(\lambda x)$ $=-2\left(d^{2} / d x^{2}\right) \log \tau_{W}(x, 0,0, \ldots)$. Thus $\mathscr{C}^{(2)}$ contains those potentials obtained directly from $W \in \mathrm{Gr}^{(2)}$ by differentiating $\log \tau_{W}$, and also scalings of those potentials. Every $u_{0} \in \mathscr{C}^{(2)}$ is a meromorphic function of $x[25, \S 5]$.

2.1. Definition. Let LP $\subset \mathscr{C}^{(2)}$ be the set of Sato-Segal-Wilson potentials $u_{0}$ which satisfy the following additional properties: (i) $u_{0}(x)$ is real and finite (i.e., no poles) for all real $x$; (ii) $L=-d^{2} / d x^{2}+u_{0}(x)$ is in the limit-point case at $x= \pm \infty$.

Fix $u_{0} \in \mathrm{LP}$, and let $m_{ \pm}(\lambda)$ be the corresponding Weyl $m$-functions. Thus

$$
m_{ \pm}(\lambda)=\phi_{ \pm}^{\prime}(0) / \phi_{ \pm}(0) \quad(\operatorname{Im} \lambda \neq 0)
$$

where $\phi_{ \pm}$are non-zero solutions of $L \phi_{ \pm}=\lambda \phi_{ \pm}$which are in $L^{2}(0, \pm \infty)$. Since these solutions are unique up to constant multiple for $\operatorname{Im} \lambda \neq 0$, the $m$-functions are well-defined. They are holomorphic, and satisfy $\operatorname{sgn}\left[\operatorname{Im} m_{ \pm}(\lambda) \cdot \operatorname{Im} \lambda\right]= \pm 1$.

Note that, with $\phi_{ \pm}(x)$ as above, the quantities $m_{ \pm}(s, \lambda)=$ $\phi_{ \pm}^{\prime}(s) / \phi_{ \pm}(s)$ are the $m$-functions for the translated potential $x \rightarrow$ $u_{0}(x+s)(s \in \mathbf{R})$.

Define

$$
\hat{\psi}(x, z)= \begin{cases}\exp \int_{0}^{x} m_{+}\left(s, z^{2}\right) d s, & \operatorname{Im} z>0, \operatorname{Re} z \neq 0, \\ \exp \int_{0}^{x} m_{-}\left(s, z^{2}\right) d s, & \operatorname{Im} z<0, \operatorname{Re} z \neq 0 .\end{cases}
$$


Then $\hat{\psi}$ is defined for all real $x$ and for all $z \in Q=\{z \in \mathbf{C} \mid \operatorname{Re} z \neq 0$, $\operatorname{Im} z \neq 0\}$. Clearly $L \hat{\psi}=z^{2} \hat{\psi}$ for all $z \in Q$, and $\hat{\psi}(0, z)=1, \hat{\psi}^{\prime}(0, z)=$ $m_{ \pm}\left(z^{2}\right)$ with the appropriate choices of sign.

It is well-known (e.g., [14, Ch. 10]) that $\left|m_{ \pm}(x, \lambda) \pm \sqrt{-\lambda}\right|=$ $O\left(|\lambda|^{-1 / 2}\right)$ as $|\lambda| \rightarrow \infty$ in closed subsectors of $\{\lambda \in \mathbf{C} \mid \operatorname{Im} \lambda \neq 0\}$. Moreover the estimate on the right is uniform (in closed subsectors) if $x$ is restricted to a compact interval. It follows that $\hat{\psi}(x, z)=$ $e^{i x z}\left(1+O\left(|\lambda|^{-1 / 2}\right)\right)$ as $|z| \rightarrow \infty$ in each closed subsector of $Q$, if $x$ is in a compact interval.

Now $u_{0}$ is $C^{\infty}$, so by, e.g. [20, pp. 37-48], $\hat{\psi}(x, z)$ has an asymptotic expansion

$$
\hat{\psi}(x, z) \sim e^{i x z}\left(1+\frac{\hat{a}_{1}(x)}{z}+\frac{\hat{a}_{2}(x)}{z^{2}}+\cdots\right),
$$

valid in $Q$. Moreover the $\hat{a}_{i}(x)$ are smooth functions which can be determined recursively by substituting $\hat{\psi}$ into $L \phi=z^{2} \phi$. Since $\hat{\psi}(0, z)=1$, we see that $\hat{a}_{i}(x)=\tilde{a}_{i}(x)$, where the $\tilde{a}_{i}$ are the coefficients of the formal Baker function (see (3)).

Since $u_{0} \in \mathscr{C}^{(2)}$, there is a true Baker function

$$
\psi(x, z)=e^{i x z}\left(1+a_{1}(x) / z+\cdots\right)
$$

which converges for large $|z|$, and which satisfies $L \psi=z^{2} \psi$. Write

$$
\psi(x, z) / \psi(0, z)=e^{i x z}\left(1+b_{1}(x) z+\cdots\right) .
$$

Using the uniqueness of $\tilde{\psi}$ in the ring $\mathscr{L}$, we see that $b_{i}(x)=\tilde{a}_{i}(x)=$ $\hat{a}_{i}(x)$ for all $i$ and $x$. Thus in each sector of $Q$, the asymptotic series $1+\hat{a}_{1}(x) / z+\cdots$ coincides with a series which converges for, say, $|z|>r$. We conclude that $\hat{\psi}(x, z)=\psi(x, z) / \psi(0, z)$ for $|z|>r$.

2.2. THEOREM. Let $u_{0}(x)$ be a real, locally-integrable function of $x \in \mathbf{R}$ such that $L=-d^{2} / d x^{2}+u_{0}(x)$ is in the limit-point case at $x= \pm \infty$. Then $u_{0} \in \mathrm{LP}$ if and only if the Weyl m-functions $m_{ \pm}(\lambda)$ have the property that

$$
\mathscr{M}(z)= \begin{cases}m_{+}\left(z^{2}\right), & \operatorname{Im} z>0, \operatorname{Re} z \neq 0, \\ m_{-}\left(z^{2}\right), & \operatorname{Im} z<0, \operatorname{Re} z \neq 0\end{cases}
$$

extends holomorphically to the region $|z|>r$ for some $r>0$. If $\mathscr{M}(z)$ admits such an extension, then $\mathscr{M}(z)$ has a simple pole at $z=\infty$ with residue $i$.

Proof. We first complete the proof of the "only if" statement. If $z \in Q$, then $\mathscr{M}(z)=\hat{\psi}^{\prime}(0, z)$ by definition of $\hat{\psi}$. Since $\hat{\psi}(x, z)$ is holomorphic in $|z|>r$ and smooth in $x$ (because $L \hat{\psi}=z^{2} \hat{\psi}$ ), we see that 
$\mathscr{M}(z)$ is holomorphic for $|z|>r$. Simple division shows that $\mathscr{M}(z)=$ $i z+\cdots$ for large $|z|$.

Let us consider the "if" statement. Suppose that $\mathscr{M}(z)$ admits an extension as described. Let $m_{ \pm}(s, z)$ correspond to $u_{0}(s+x)$, and let $\mathscr{M}(s, z)$ be defined by (4) with $m_{ \pm}\left(s, z^{2}\right)$ in place of $m_{ \pm}\left(z^{2}\right)$. Then $\mathscr{M}(s, z)$ is holomorphic in $|z|>r_{1}$ for each $s \in \mathbf{R}$, and is jointly continuous in $s \in \mathbf{R}$ and $|z|>r_{1}$. Here $r_{1} \geq r$ is independent of $s$.

We prove the last statement. First recall that $\operatorname{sgn}\left[\operatorname{Im} m_{ \pm}(s, \lambda) \cdot \operatorname{Im} \lambda\right]$ $= \pm 1$ if $\operatorname{Im} \lambda \neq 0$. Note also that $\mathscr{M}(s, z)$ is meromorphic in $|z|>r$. These facts imply that $\mathscr{M}(s, z)$ takes values in $\mathbf{R} \cup\{\infty\}$ if and only if $z$ is pure imaginary, i.e., if and only if $\lambda=z^{2} \leq-r^{2}$.

Next note that, for fixed $s, m_{-}(s, \lambda)$ increases and $m_{+}(s, \lambda)$ decreases as $\lambda \downarrow-\infty$ (unless $\lambda$ is a pole, of course). Now, $\mathscr{M}(z)$ has no poles for $|z|>r$. Thus we can find $r_{1} \geq r$ such that, if $\lambda \leq-r_{1}^{2}$, then $m_{-}(0, \lambda)$ and $m_{+}(0, \lambda)$ are never equal. It follows that, if $s \in \mathbf{R}$ and $\lambda \leq-r_{1}^{2}$, then $m_{-}(s, \lambda)$ and $m_{+}(s, \lambda)$ are never equal. This implies that $\mathscr{M}(s, z)$ omits some interval of real values on $|z|>r_{1}$. By the Picard theorem [2], $\mathscr{M}(s, z)$ is meromorphic at $z=\infty$. By the preceding paragraph, $\mathscr{M}(s, z)$ has at most a simple pole at $z=\infty$, and by the relations $\mid m_{ \pm}(s, \lambda)$ $\pm \sqrt{-\lambda} \mid \rightarrow 0$ if $|\lambda| \rightarrow \infty$ with $\delta<|\arg \lambda|<\pi-\delta([14])$, we see that $\mathscr{M}(s, z)=i z+\cdots$. It follows from this and the first sentence of the present paragraph that $\mathscr{M}(s, z)$ is holomorphic for $|z|>r_{1}$. The continuity statement is clear.

Define

$$
\hat{\psi}(x, z)=\exp \int_{0}^{x} \mathscr{M}(s, z) d s \quad\left(|z|>r_{1}\right) .
$$

We can write

$$
\hat{\psi}(x, z)=e^{i x z}\left(1+\frac{\hat{a}_{1}(x)}{z}+\frac{\hat{a}_{2}(x)}{z^{2}}+\cdots\right) \quad(x \in \mathbf{R}),
$$

where the series converges for $|z|>r_{1}$ and the coefficients are continuously differentiable for $x$. In fact they are obtained by integrating the coefficients of $\mathscr{M}(s, z)$ and combining powers of $1 / z$ in the exponential; this can be proved using the Montel theorem [2].

We now follow Segal-Wilson [25, Prop. 5.22 and the preceding discussion]. First of all, we scale $u_{0}$ (i.e., replace $u_{0}$ by $\delta^{2} u_{0}(\delta x)$ for sufficiently small $\delta>0)$ so as to make $\mathscr{M}(z)$ holomorphic in $|z|>1-\varepsilon$ for some $\varepsilon>0$. Consider the closed subspace $W \subset L^{2}(\mathbf{K})$ which contains $1=\hat{\psi}(0, z), \mathscr{M}(z)=\hat{\psi}^{\prime}(0, z)$, and is invariant under multiplication by $z^{2}$. Then $W \in \mathrm{Gr}^{(2)}$ [25], and $W$ is transverse by its very definition, i.e., 
contains no function whose Laurent expansion about $z=0$ consists entirely of negative powers of $z$.

Next let $\phi_{i}\left(x, z^{2}\right)$ be the solutions of $L \phi=z^{2} \phi$ satisfying $D^{j} \phi_{i}\left(0, z^{2}\right)$ $=\delta_{i j}(i, j=1,2)$. Then the $\phi_{i}$ are entire in $z^{2}$ for each $x \in \mathbf{R}$. Also, $\hat{\psi}(x, z)$ and $\phi_{1}(x, z) \hat{\psi}(0, z)+\phi_{2}(x, z) \hat{\psi}^{\prime}(0, z)$ are both solutions of $L \phi=$ $z^{2} \phi$ with the same initial conditions, hence are equal for all $x \in \mathbf{R}$. Since $W$ is $z^{2}$-invariant, it follows that $\hat{\psi}(x, z) \in W$ for all $x \in \mathbf{R}$. Moreover $\hat{\psi}(x, z)=e^{i x z}(1+$ lower order terms in $z)$ for each $x$. However, these two properties characterize the Baker function $\psi_{W}(x, z)$, at least if $\exp (-i x z) \cdot$ $W$ is transverse; see the beginning of this section and [25, Prop. 5.1]. Let $u_{W}(x)$ be the potential in $\mathscr{C}^{(2)}$ defined by $W$. Then $u_{W}$ is meromorphic in $x[25, \S 5]$. Thus $\exp (-i x z) \cdot W$ is transverse except for isolated points (the poles of $\left.u_{W}\right)$, and we conclude that $\hat{\psi}(x, z)=\psi_{W}(x, z)$ except perhaps at these poles. But since $u_{0}$ is locally integrable, there are no poles. Thus $u_{0}=u_{W} \in \mathrm{LP}$, which is what we wanted to prove. This completes the proof of Theorem 2.2.

We finish the section by using a simple limit procedure to construct potentials in LP. First consider a quasi-periodic potential $u$ of algebro-geometric type $[11,18,21]$. Thus the spectrum $\Sigma$ of $L=-d^{2} / d x^{2}+u(x)$ (viewed as a self-adjoint operator on $L^{2}(-\infty, \infty)$ ) is a finite union of intervals: $\Sigma=\left[\lambda_{0}, \lambda_{1}\right] \cup\left[\lambda_{2}, \lambda_{3}\right] \cup \cdots \cup\left[\lambda_{2 g}, \infty\right)$. Moreover one has

$$
u(x)=\sum_{i=0}^{2 g} \lambda_{\imath}-2 \sum_{j=1}^{g} P_{j}(x)
$$

where $P_{j}(x) \in\left[\lambda_{2 j-1}, \lambda_{2 j}\right](1 \leq j \leq g)$ and the motion of $P_{j}$ is determined by

$$
P_{j}^{\prime}=\left.\frac{ \pm \sqrt{\left(\lambda-\lambda_{0}\right)\left(\lambda-\lambda_{1}\right) \cdots\left(\lambda-\lambda_{2 g}\right)}}{\prod_{s \neq j}\left(P_{j}-P_{s}\right)}\right|_{\lambda=P_{j}} \quad(1 \leq j \leq g) .
$$

See $[\mathbf{1 8}, \mathbf{2 1}]$.

Let us now choose a sequence $\left\{u_{n}\right\}_{n=1}^{\infty}$ of such potentials in the following way. Let $\Sigma_{n}$ be the spectrum of $L_{n}=-d^{2} / d x^{2}+u_{n}(x)$ as a self-adjoint operator on $L^{2}(-\infty, \infty)$. We suppose that $-r^{2}<\lambda_{0}^{(n)}<\lambda_{2 g}^{(n)}=$ $r^{2}$ for some $r>0$ independent of $n$. Further we suppose that $\Sigma_{n+1} \subset \Sigma_{n}$, that $C=\left(-\infty, r^{2}\right) \cap \cap_{n=1}^{\infty} \Sigma_{n}$ is a Cantor set, and that $u_{n}(x)$ converges to a limit function $u_{0}(x)$, uniformly on compact subsets of $\mathbf{R}$. It is clear from (5) and (6) that such a sequence can be found. Note that $\left|u_{n}(x)\right| \leq$ $2 r^{2}(x \in \mathbf{R}, n=0,1,2, \ldots)$.

It is easy to check that the spectrum $\Sigma_{0}$ of $L_{0}=-d^{2} / d x^{2}+u_{0}(x)$ equals $C \cup\left[r^{2}, \infty\right)$ (this uses the fact that $\Sigma_{n}$ decreases with $n$ ). That is, $\Sigma_{0}$ has a "Cantor-like part". 
It must be shown that $u_{0} \in$ LP. Let $m_{ \pm}^{(n)}(\lambda)$ be the $m$-functions for $L_{n}$, and let $\mathscr{M}_{n}(z)$ be the function defined by (4) $(n=0,1,2, \ldots)$. It follows from [11] (see also [10]) that $\mathscr{M}_{n}(z)$ extends holomorphically to $|z|>r(n \geq 1)$. It can also be shown that there is a fixed interval $I \subset \mathbf{R}$ such that $\left\{m_{+}^{(n)}(\lambda) \mid \lambda \leq-4 r^{2}\right\} \cup\left\{m_{-}^{(n)}(\lambda) \mid \lambda \leq-4 r^{2}\right\}$ does not intersect $I$ for larger $n$. This assertion follows from the convergence $u_{n} \rightarrow u_{0}$ and the bound $\left\|u_{n}\right\|_{\infty} \leq 2 r^{2}(n \geq 0)$; we omit the proof.

We conclude that each $\mathscr{M}_{n}(z)$ omits the set $I$ of values for $|z|>2 r$ $(n=1,2, \ldots)$. By the Montel theorem [2], $\left\{\mathscr{M}_{n}\right\}_{n=1}^{\infty}$ is a normal family of holomorphic functions on $\{z|| z \mid>2 r\}$. One checks that $m_{ \pm}^{(n)}(\lambda) \rightarrow$ $m_{ \pm}^{(0)}(\lambda)$ if $\operatorname{Im} \lambda \neq 0$. Hence $\tilde{\mathscr{M}}_{0}(z)=\lim _{n \rightarrow \infty} \mathscr{M}_{n}(z)$ is well-defined and equals $\mathscr{M}_{0}(z)$ for $z \in Q,|z|>2 r$. By Theorem 2.2, $u_{0} \in$ LP.

2.3. Remarks (a). It seems unlikely that the above procedure will always produce an almost periodic $u_{0}$. However, using the more detailed construction of Chulaevsky [4] one can obtain limit-periodic potentials which are in LP.

(b) Neither the construction above nor that of [4] make it clear that the resulting potential is meromorphic in the complex $x$-plane. This is a remarkable consequence of the Segal-Wilson theory.

3. The Floquet exponent. In this section we will describe a method for finding potentials in the class LP which generalizes the one given at the end of $\S 2$. We will use the Floquet exponent $w=w(\lambda)$ of $-d^{2} / d x^{2}+$ $u_{0}(x)[10,15,16]$. This quantity is defined with respect to a "stationary ergodic process" of potentials, and not just with respect to a single $u_{0}$. For our purposes, it is convenient to adopt the following definitions [17].

3.1. Definitions. Let $\Omega=L_{\text {real }}^{2}\left(\mathbf{R},\left(1+|x|^{3}\right)^{-1} d x\right)$ with the Borel field $\mathscr{B}$ defined by the weak topology. Let $\left\{\tau_{s} \mid s \in \mathbf{R}\right\}$ be the shift operators defined by $\left(\tau_{s} u\right)(x)=u(s+x)(u \in \Omega, s \in \mathbf{R})$. Let $\mu$ be a probability measure on $(\Omega, \mathscr{B})$ such that $\mu$ restricted to each ball $\left\{u \mid\|u\|_{\Omega}\right.$ $\leq R\}$ is Radon, and such that

$$
\begin{aligned}
& \mu\left(\tau_{x}(A)\right)=\mu(A) \text { for all } x \in \mathbf{R}, A \in \mathscr{B} ; \\
& \int_{\Omega}\left(\int_{0}^{1}|u(s)|^{2} d s\right) d \mu(u)<\infty
\end{aligned}
$$

Then $(\Omega, \mathscr{B}, \mu)$ is a stationary stochastic process, and $\mu$ is invariant. If in addition:

$$
\mu\left(\tau_{x}(A) \Delta A\right)=0 \text { for all } x \in \mathbf{R} \rightarrow \mu(A)=0 \text { or } 1
$$


for each $A \in \mathscr{B}$, then $(\Omega, \mathscr{B}, \mu)$ is a stationary ergodic process, and $\mu$ is ergodic.

Kotani [17] shows that any $u \in \Omega$ is in the limit-point case at $x= \pm \infty$. Let $m_{ \pm}(\lambda) \equiv m_{ \pm}(u, \lambda)$ be the Weyl $m$-functions; they are holomorphic in $\lambda$ for $\operatorname{Im} \lambda \neq 0$, and jointly continuous in $(u, \lambda)$ when $\Omega$ has the weak topology.

Let $(\Omega, \mathscr{B}, \mu)$ be a stationary stochastic process. Define

$$
w(\lambda)=w_{\mu}(\lambda)=\int_{\Omega} m_{+}(u, \lambda) d \mu(u) .
$$

Since $u \rightarrow m_{+}(u, \lambda)$ is $\mu$-integrable [17], this definition makes sense. One can show that $w(\lambda)$ is holomorphic in the upper half-plane $U=\{\lambda \in$ $\mathbf{C} \mid \operatorname{Im} \lambda>0\}$. Moreover $\operatorname{Im} w>0, \operatorname{Re} w<0$, and $\operatorname{Im} d w / d \lambda>0$ for $\lambda \in$ $U$. If $\mu$ is ergodic, then $w$ has additional properties which justify the name "Floquet exponent". Especially, the boundary value

$$
\hat{w}(\lambda)=\beta(\lambda)+i \alpha(\lambda)=\lim _{\varepsilon \rightarrow 0^{+}} w(\lambda+i \varepsilon) \quad(\lambda \in \mathbf{R})
$$

satisfies the following conditions. (i) The rotation number $\lambda \rightarrow \alpha(\lambda)=$ $\lim _{x \rightarrow \infty} 1 / x \arg \left(\phi^{\prime}(x)+i \phi(x)\right)$ is continuous, monotone increasing, and increases exactly on the spectrum $\Sigma_{u}$ of $L_{u}=-d^{2} / d x^{2}+u(x)$ for $\mu$ - a.a. $u$ ([15]; see also [16]). (ii) The Lyapunov number $\beta(\lambda)=$ $\lim _{x \rightarrow \infty}(1 / 2 x) \ln \left[\phi^{2}(x)+\phi^{\prime 2}(x)\right]$ determines the absolutely continuous spectrum $\sum_{u}^{a c}$ of $L_{u}$ for $\mu$ - a.e. $u$; in fact the essential support of $\sum_{u}^{a c}$ is $\{\lambda \in \mathbf{R} \mid \beta(\lambda)=0\}[\mathbf{1 6}]$.

Kotani proves the following result [17].

3.2. THEOREM. Suppose $w=w(\lambda)$ is a holomorphic function on $U$ such that $\operatorname{Im} w>0, \operatorname{Re} w<0$, and $\operatorname{Im}(d w / d \lambda)>0$ for $\lambda \in U$. Suppose in addition that $\lim _{\lambda \rightarrow-\infty} w(\lambda) / \sqrt{-\lambda}=1$, and that there exists $r^{2}>0$ such that $\beta(\lambda)<0$ for $\lambda \leq 0$ and $\beta(\lambda)=0$ for $\lambda \geq r^{2}$. Then there is $a$ stationary stochastic process $(\Omega, \mathscr{B}, \mu)$ such that: (i) $w=w_{\mu}$; (ii) $\mu\{u \in$ $\Omega \mid\left\langle L_{u} \phi, \psi\right\rangle$ is non-negative definite as a bilinear form on $\left.C_{\text {compact }}^{\infty}(\mathbf{R})\right\}=1$.

We will also use the following theorem of De Concini-Johnson [10]. Though their result is stated for a slightly different space $\Omega$, the proof works in the case at hand.

3.3. THEOREM. Let $(\Omega, \mathscr{B}, \mu)$ be a stationary ergodic process such that $\Omega$ is (weakly) compact, and such that the topological support of $\mu$ equals $\Omega$. Let $w=w_{\mu}$ be the corresponding Floquet exponent. 
(a) Suppose that $\beta(\lambda)=0$ for a.a. $\lambda$ in an open interval $I \subset \mathbf{R}$. Then for each $u \in \Omega$ : the function $\lambda \rightarrow m_{+}(u, \lambda)$ extends holomorphically from $U$ through I, and the extended function equals $m_{-}(u, \lambda)$ for $\operatorname{Im} \lambda<0$. The same statement holds with + and - interchanged.

(b) Suppose the spectrum $\Sigma=\Sigma_{u}$ of $L_{u}$ is a finite union of intervals for $\mu$-a.a. $u \in \Omega$, and that $\beta(\lambda)=0$ for a.a. $\lambda \in \Sigma$. Then each $u \in \Omega$ is an algebro-geometric potential ( see $\S 2$ ).

We now turn to the main result of this section.

3.4. TheOREM. Let $w=w(\lambda)$ satisfy the conditions of Theorem 3.2. Then there is a stationary ergodic process $(\Omega, \mathscr{B}, \mu)$ which satisfies (i) and (ii) of 3.2 such that $u \in \mathrm{LP}$ for $\mu$-a.a. $u \in \Omega$.

Our proof of 3.4 repeats a good share of Kotani's proof of 3.2.

Proof. Following Kotani, we construct potentials $u_{k}(k \geq 1)$ with the following properties. (i) The function $u_{k}(x)$ is $T_{k}$-periodic and belongs to $\Omega$ (i.e., is in $L^{2}\left[0, T_{k}\right]$ ). (ii) The Floquet exponent $w_{k}$ (defined by normalized Haar measure $\mu_{k}$ on the circle $\left.C_{k}=\left\{\tau_{s} u_{k} \mid 0 \leq s \leq T_{k}\right\} \subset \Omega\right)$ satisfies $\beta_{k}(\lambda)=\operatorname{Re} w_{k}(\lambda)=0$ for $\lambda \geq r_{k}^{2}$, where $r_{k} \rightarrow r$ as $k \rightarrow \infty$. (iii) $\beta_{k}(\lambda)>0$ for $\lambda \leq 0$. (iv) $w_{k}(\lambda) \rightarrow w(\lambda)$, uniformly on compact subsets of $U$.

Condition (ii) implies that the spectrum $\Sigma_{k}$ of $L_{k}=-d^{2} / d x^{2}+u_{k}(x)$ contains $\left[r_{k}^{2}, \infty\right)$; also, (iii) implies that $\Sigma_{k} \subset(0, \infty)$, since $u_{k}$ is periodic (see, e.g., Moser [19, Ch. 3]). Again by periodicity of $u_{t}, \Sigma_{k}$ is a finite union of intervals, and $\beta_{k}(\lambda)=0$ for all $\lambda \in \Sigma_{k}$. By Theorem 3.3, $u_{k}(x)$ is an algebro-geometric potential. Thus from (5) in $\S 2$,

$$
u_{k}(x)=\sum_{i=0}^{2 g_{k}} \lambda_{l}^{(k)}-2 \sum_{j=1}^{g_{k}} P_{j}^{(k)}(x) \text {, }
$$

where

$$
P_{j}^{(k)}(x) \in\left[\lambda_{2 j-1}^{(k)}, \lambda_{2 j}^{(k)}\right] \text { and } 0<\lambda_{0}^{(k)}<\cdots<\lambda_{2 g_{k}}^{(k)} \leq r_{k}^{2} .
$$

We conclude that $\left|u_{k}(x)\right| \leq 2 r_{k}^{2}<2\left(r^{2}+1\right)$ for all large $k$.

The circles $C_{k}$ are thus all contained in the weakly compact and translation-invariant subset $\Omega_{1}=\operatorname{cls}\left\{u \mid\|u\|_{\infty} \leq 2\left(r^{2}+1\right)\right\} \subset \Omega$. The measures $\mu_{k}$ define Radon measures on $\Omega_{1}$, hence there is a weak limit point $\mu$ of $\left\{\mu_{k}\right\}_{k=1}^{\infty}$. The topological support $\Omega_{\mu}$ of $\mu$ is contained in $\Omega_{1}$. Since the translations $\left\{\tau_{x} \mid x \in \mathbf{R}\right\}$ are weakly continuous on $\Omega_{1}, \mu$ is invariant. Also $w=w_{\mu}$ by weak continuity of $u \rightarrow m_{+}(u, \lambda)$. 
Next introduce an ergodic decomposition [22] $\left\{\mu_{\gamma} \mid \gamma \in \Gamma\right\}$ of $\mu$. Thus $\Gamma$ is a measure space with probability measure $\sigma$, each $\mu_{\gamma}$ is an ergodic measure on $\Omega_{\mu} \subset \Omega$, and for all continuous functions $h: \Omega \rightarrow \mathbf{R}$ one has

$$
\int_{\Omega} h d \mu=\int_{\Gamma}\left(\int_{\Omega} h d \mu_{\gamma}\right) d \sigma(\gamma)
$$

In particular, letting $w_{\gamma}(\lambda)$ be the Floquet exponent with respect to $\mu_{\gamma}$, one has

$$
w_{\mu}(\lambda)=\int_{\Gamma} w_{\gamma}(\lambda) d \sigma(\gamma) \quad(\operatorname{Im} \lambda>0) .
$$

Let $K \subset U$ be precompact in cls $U$ (i.e., $K$ is a bounded subset of $U)$. Then there is a constant $c_{K}$ depending only on $K$ such that $\left|\operatorname{Re} w_{\gamma}(\lambda)\right| \leq c_{K}$ for all $\gamma \in \Gamma$ and $\lambda \in K$. This follows from the description of $\beta_{\gamma}(\lambda)$ as a Lyapunov number, together with the estimates of [17, Lemma 2.8]. Let $R=r^{2}$, and let $n \geq 2$. By bounded convergence we have

$$
\begin{aligned}
0 & =\int_{R}^{n R} \operatorname{Re} w_{\mu}(\lambda) d \lambda=\lim _{\varepsilon \rightarrow 0^{+}} \int_{R}^{n R} \operatorname{Re} w_{\mu}(\lambda+i \varepsilon) d \lambda \\
& =\lim _{\varepsilon \rightarrow 0^{+}} \int_{R}^{n R} \int_{\Gamma} \operatorname{Re} w_{\gamma}(\lambda+i \varepsilon) d \sigma(\gamma) d \lambda \\
& =\int_{\Gamma} \lim _{\varepsilon \rightarrow 0^{+}} \int_{R}^{n R} \operatorname{Re} w_{\gamma}(\lambda+i \varepsilon) d \lambda .
\end{aligned}
$$

We conclude that, for $\sigma$-a.a. $\gamma, \beta_{\gamma}(\lambda)=\operatorname{Re} w_{\gamma}(\lambda)=0$ for a.a. $\lambda \geq R=r^{2}$.

Now use Theorem 3.3(a): for each $u$ in the support of $\mu_{\gamma}, \lambda \rightarrow$ $m_{ \pm}(u, \lambda)$ extends holomorphically from the upper half-plane $U$ through $\left(r^{\frac{ \pm}{2}}, \infty\right)$, and the extension equals $m_{\mp}(u, \lambda)$ in the lower half-plane.

Next consider $L_{u}=-d^{2} / d x^{2}+u(x)$ with domain $\mathscr{D}=C_{\text {compact }}^{\infty}(\mathbf{R}) \subset$ $L^{2}(\mathbf{R})$. Since $L_{u}$ is in the limit-point case at $x= \pm \infty$, it has deficiency indices zero, hence has a unique self-adjoint extension (its closure), which moreover is associated to the non-negative bilinear form $\left\langle L_{u} \phi, \psi\right\rangle$ on $\mathscr{D}$ [12]. Therefore this self-adjoint extension has no spectrum in $(-\infty, 0)$. One now proves in a standard way that $m_{ \pm}(u, \lambda)$ are meromorphic on $\operatorname{Re} \lambda<0$, and that $m_{-}(u, \lambda) \neq m_{+}(u, \lambda)$ there. Since $m_{+}(u, \lambda)$ decreases and $m_{-}(u, \lambda)$ increases as $\lambda \downarrow-\infty$, we can find $r_{1} \geq r$ such that $\mathscr{M}(z)=$ $\mathscr{M}(u, z)$ has no poles on $|z|>r_{1}$, i.e., is holomorphic there. By Theorem 2.2, $u \in$ LP. Note that $\mathscr{M}(z)=i z+\cdots$ for large $|z|$; therefore $\mathscr{M}(z)$ is holomorphic for $\operatorname{Re} z^{2}=\operatorname{Re} \lambda<0$. Hence $\mathscr{M}(z)$ is holomorphic on $|z|>$ $r$.

Finally, let $u \in \Omega_{\mu}$. We can find $u_{n}$ in $\Omega_{\mu}$ such that $u_{n} \rightarrow u$ weakly and such that each $u_{n}$ is in the support of some $\mu_{\gamma_{n}}$. The $m$-functions 
$m_{ \pm}\left(u_{n}, \lambda\right)$ are meromorphic on $\operatorname{Re} \lambda<0$, and $m_{+}\left(u_{n}, \lambda\right)<m_{-}\left(u_{n}, \lambda\right)$ for negative real $\lambda$. Furthermore $m_{+}\left(u_{n}, \lambda\right)$ decreases and $m_{-}\left(u_{n}, \lambda\right)$ increases as $\lambda \downarrow-\infty$. Choosing a subsequence if necessary, we can assume that $m_{ \pm}\left(u_{n},-r^{2}\right)$ are convergent sequences in $\mathbf{R} \cup\{\infty\}$. Then for large $n,\left\{m_{+}\left(u_{n}, \lambda\right) \mid \operatorname{Re} \lambda<-r^{2}\right\}$ and $\left\{m_{-}\left(u_{n}, \lambda\right) \mid \operatorname{Re} \lambda<-r^{2}\right\}$ omit intervals $I_{ \pm}$of real values. Using the Montel theorem once again, we see that $\left\{m_{+}\left(u_{n}, \cdot\right) \mid n \geq 1\right\}$ and $\left\{m_{-}\left(u_{n}, \cdot\right) \mid n \geq 1\right\}$ are normal families of meromorphic functions for $\operatorname{Re} \lambda<-r^{2}$. Using the weak continuity in $u$ of $m_{ \pm}(u, \lambda)$ for $\operatorname{Im} \lambda \neq 0$, we conclude easily that $\mathscr{M}\left(u_{n}, z\right) \rightarrow \mathscr{M}(u, z)$ for $|z|>r$, and that $\mathscr{M}(z)=i z+\cdots$. Thus $\mathscr{M}(z)$ is holomorphic on $|z|>r$, and so $u \in$ LP by Theorem 2.2.

3.5. Remarks (a). We have actually shown that $u \in$ LP for all $u$ in the topological support $\Omega_{\mu}$ of $\Omega$.

(b) One can replace the assumption $\operatorname{Re} w(\lambda)<0$ for $\lambda \leq 0$ by $\operatorname{Re} w(\lambda)<0$ for $\operatorname{Re} \lambda \leq c$, for any constant $c<r^{2}$.

(c) Let $(\Omega, \mathscr{B}, \mu)$ be a stationary ergodic process such that the topological support $\Omega_{\mu}$ of $\mu$ is compact. Suppose further that there is a fixed constant $r$ such that: (i) the operators $L_{u}$ satisfy $\left\langle L_{u} \phi, \phi\right\rangle \geq-r^{2}\langle\phi, \phi\rangle$ for all smooth $\phi$ with compact support; (ii) $\operatorname{Re} w(\lambda)=0$ for $\lambda \geq r^{2}$. Then from the proof of 3.4 one sees that $u \in \operatorname{LP}$ for each $u \in \Omega_{\mu}$.

(d) The point of 3.2 is that the function $w(\lambda)$ is quite general. One can, for example, choose $w(\lambda)$ so that $\lim _{\varepsilon \rightarrow 0^{+}} \operatorname{Re} w(\lambda)=\beta(\lambda)<0$ for all $\lambda<r^{2}$. Then either $\Omega$ contains only the constant function $u(x) \equiv r^{2}$, or $\mu$-a.a. $u \in \Omega$ have spectrum in $\left(-\infty, r^{2}\right)$ ([16]; also [10]). Only the latter possibility is of interest. It indicates (but does not prove) that there exist $u \in \mathrm{LP}$ with at least some point spectrum.

\section{REFERENCES}

[1] F. Calogero, and A. Degasperis, Spectral Transform and Solitons I, North-Holland, Amsterdam, 1982.

[2] C. Caratheodory, Funktionentheorie II, zweite Auflage, Birkhäuser Verlag, Basel und Stuttgart, 1961.

[3] E. Coddington, and N. Levinson, Theory of Ordinary Differential Equations, McGraw-Hill, New York, 1955.

[4] V. Chulaevsky, Inverse Spectral Problem for Limit-Periodic Schrödinger Operators, Functional Anal. Appl., 18 (1984), 230-233.

[5] E. Date, M. Kashiwara, M. Jimbo, and T. Miwa, The $\tau$-function of the KadomtsevPetviashvili equation, Proc. Japan Acad., 57A (1981), 342-347.

[6] E. Date, et al., Vertex operators and $\tau$-functions, Proc. Japan Acad., 57A (1981), 387-392.

[7] Operator approach to the Kadomtsev-Petviashvili equation, J. Phys. Soc. Japan, 50 (1981), 3806-3812. 

, Transformation groups for Soliton equations, Publ. Res. Inst. Math. Sci., Kyoto, 18 (1982), 1077-1110.

[9] Transformation groups for Soliton equations, Proc. Res. Inst. Math. Sci., Symposium on Non-Linear Integrable Systems, May 1981, pp. 39-119.

[10] C. De Concini, and R. Johnson, The algebraic-geometric AKNS potentials, J. Ergodic Theory and Dyn. Sys., 7 (1987), 1-24.

[11] V. Dubrovin, S. Novikov, and V. Matveev, Non-linear equations of Korteweg-de Vries type, finite-zone linear operators, and Abelian varieties, Russian Math. Surveys, 31 (1976), 59-146.

[12] N. Dunford, and J. Schwarz, Linear Operators, Vol. II, Interscience, New York, London, 1963.

[13] R. Giachetti, and R. Johnson, The Floquet exponent for two-dimensional linear systems with bounded coefficients, J. Math. Pures Appl., 65 (1986), 93-117.

[14] E. Hille, Lectures on Ordinary Differential Equations, Addison-Wesley, Reading, Mass., 1969.

[15] R. Johnson, and J. Moser, The rotation number for almost periodic potentials, Comm. Math. Phys., 84 (1982), 403-438.

[16] S. Kotani, Lyapounov Indices Determine Absolutely Continuous Spectra of Stationary Random One-dimensional Schrödinger Operators, Taniguchi Symp. SA, Katata, (1982), 225-247.

[17] _ On an Inverse Problem for Random Schrödinger Operators, to appear in Springer Conference Proceedings, 1985.

[18] H. McKean, and P. Van Moerbeke, The spectrum of Hills' equation, Invent. Math., 30 (1975), 217-274.

[19] J. Moser, Integrable Hamiltonian Systems and Spectral Theory, Lezioni Fermiane, Pisa, 1981.

[20] M. Naimark, Lineare Differentialoperatoren, Akademie-Verlag, Berlin, 1960.

[21] S. Novikov, The Periodic Korteweg-de Vries Problem, Functional Anal. Appl, 8 (1974), 54-66.

[22] R. Phelps, Lectures on Choquet's Theorem, Van Nostrand Math. Studies, American Book Co., New York, 1966.

[23] M. Sato, Soliton equations as dynamical systems on infinite-dimensional Grassmann manifolds, Publ. Res. Inst. Math. Sci., Kokyuroku, 439 (1981), 30.

[24] M. Sato, and Y. Sato, On Hirota's Bilinear Equations I, II, Publ. Res. Inst. Math. Sci., Kokyuroku, 388 (1980), 183; 414 (1981), 181.

[25] G. Segal, and G. Wilson, Loop groups and equations of KdV type, Publ. IHES, 61 (1985), 5-65.

[26] H. Weyl, Über gewöhnliche lineare Differentialgleichungen mit Singularitäten und die zugehörigen Entwicklungen willkürlicher Funktionen, Math. Annalen, 68 (1910), 220-269.

Received January 26, 1987.

SONDERFORSCHUNGSBEREICH 123

UNIVERSITÄT HEIDELBERG

D-6900 HEIDELBERG, BRD

AND

UNIVERSITY OF SOUTHERN CALIFORNIA

LOS ANGELES, CA 90089-1113 



\title{
PACIFIC JOURNAL OF MATHEMATICS EDITORS
}

\author{
V. S. VARADARAJAN \\ (Managing Editor) \\ University of California \\ Los Angeles, CA 90024 \\ HERBERT Clemens \\ University of Utah \\ Salt Lake City, UT 84112 \\ R. FINN \\ Stanford University \\ Stanford, CA 94305
}

\author{
HERMANN FLASCHKA \\ University of Arizona \\ Tucson, AZ 85721
}

Ramesh A. Gangolli University of Washington Seattle, WA 98195

VAUGHAN F. R. JONES University of California Berkeley, CA 94720
ROBION KIRBY

University of California

Berkeley, CA 94720

C. C. MOORE

University of California

Berkeley, CA 94720

HAROLD STARK

University of California, San Diego La Jolla, CA 92093

\section{ASSOCIATE EDITORS}

\author{
R. ARENS \\ E. F. BECKENBACH \\ B. H. NEUMANN \\ F. WOLF \\ K. YOSHIDA \\ (1906-1982)

\section{SUPPORTING INSTITUTIONS}

\section{UNIVERSITY OF ARIZONA} \\ UNIVERSITY OF BRITISH COLUMBIA \\ CALIFORNIA INSTITUTE OF TECHNOLOGY \\ UNIVERSITY OF CALIFORNIA \\ MONTANA STATE UNIVERSITY \\ UNIVERSITY OF NEVADA, RENO \\ NEW MEXICO STATE UNIVERSITY \\ OREGON STATE UNIVERSITY \\ UNIVERSITY OF OREGON \\ UNIVERSITY OF SOUTHERN CALIFORNIA \\ STANFORD UNIVERSITY \\ UNIVERSITY OF HAWAII \\ UNIVERSITY OF TOKYO \\ UNIVERSITY OF UTAH \\ WASHINGTON STATE UNIVERSITY \\ UNIVERSITY OF WASHINGTON
}

The Supporting Institutions listed above contribute to the cost of publication of this Journal, but they are not owners or publishers and have no responsibility for its content or policies.

Mathematical papers intended for publication in the Pacific Journal of Mathematics should be in typed form or offset-reproduced (not dittoed), double spaced with large margins. Please do not use built up fractions in the text of the manuscript. However, you may use them in the displayed equations. Underline Greek letters in red, German in green, and script in blue. The first paragraph must be capable of being used separately as a synopsis of the entire paper. In particular it should contain no bibliographic references. Please propose a heading for the odd numbered pages of less than 35 characters. Manuscripts, in triplicate, may be sent to any one of the editors. Please classify according to the scheme of Math. Reviews, Index to Vol. 39. Supply name and address of author to whom proofs should be sent. All other communications should be addressed to the managing editor, or Elaine Barth, University of California, Los Angeles, California 90024

There are page-charges associated with articles appearing in the Pacific Journal of Mathematics. These charges are expected to be paid by the author's University, Government Agency or Company. If the author or authors do not have access to such Institutional support these charges are waived. Single authors will receive 50 free reprints; joint authors will receive a total of 100 free reprints. Additional copies may be obtained at cost in multiples of 50 .

The Pacific Journal of Mathematics is issued monthly as of January 1966. Regular subscription rate: $\$ 190.00$ a year (5 Vols., 10 issues). Special rate: $\$ 95.00$ a year to individual members of supporting institutions.

Subscriptions, orders for numbers issued in the last three calendar years, and changes of address should be sent to Pacific Journal of Mathematics, P.O. Box 969, Carmel Valley, CA 93924, U.S.A. Old back numbers obtainable from Kraus Periodicals Co., Route 100, Millwood, NY 10546.

The Pacific Journal of Mathematics at P.O. Box 969, Carmel Valley, CA 93924 (ISSN 0030-8730) publishes 5 volumes per year. Application to mail at Second-class postage rates is pending at Carmel Valley, California, and additional mailing offices. Postmaster: send address changes to Pacific Journal of Mathematics, P.O. Box 969, Carmel Valley, CA 93924.

PUBLISHED BY PACIFIC JOURNAL OF MATHEMATICS, A NON-PROFIT CORPORATION Copyright (C) 1988 by Pacific Journal of Mathematics 


\section{Pacific Journal of Mathematics}

\section{Vol. 132, No. $2 \quad$ February, 1988}

Jeffery Marc Bergen and Luisa Carini, A note on derivations with power central values on a Lie ideal ..............................209

Alfonso Castro and Sumalee Unsurangsie, A semilinear wave equation

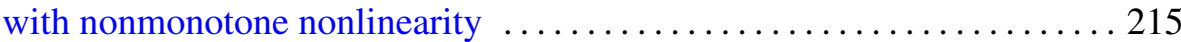

Marius Dadarlat, On homomorphisms of matrix algebras of continuous

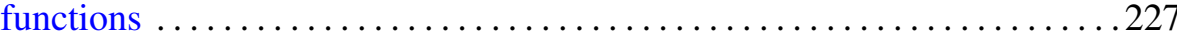

A. Didierjean, Quelques classes de cobordisme non orienté refusant de se

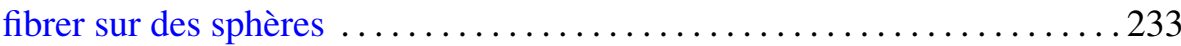

Edward George Effros and Zhong-Jin Ruan, On matricially normed spaces

Sherif El-Helaly and Taqdir Husain, Orthogonal bases are Schauder bases

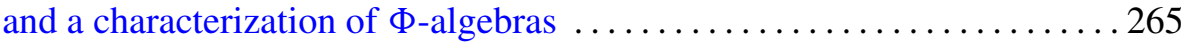

Edward Richard Fadell and Peter N-S Wong, On deforming $G$-maps to be

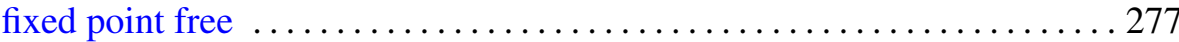

Jean-Jacques Gervais, Stability of unfoldings in the context of equivariant

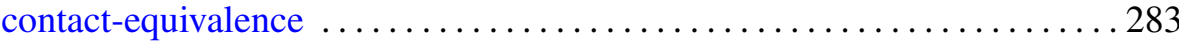

Douglas Martin Grenier, Fundamental domains for the general linear group

Ronald Scott Irving and Brad Shelton, Loewy series and simple projective

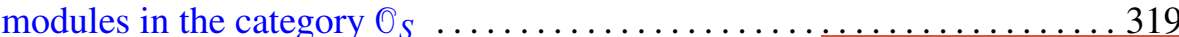

Russell Allan Johnson, On the Sato-Segal-Wilson solutions of the K-dV equation

Thomas Alan Keagy and William F. Ford, Acceleration by subsequence transformations

Min Ho Lee, Mixed cusp forms and holomorphic forms on elliptic varieties

Charles Livingston, Indecomposable surfaces in 4-space 